\title{
Markovian Queueing Model for Throughput Maximization in D2D-Enabled Cellular Networks
}

\author{
Abiodun Gbenga-Ilori ${ }^{1}$ and Olufunmilayo Sanusi ${ }^{2}$ \\ ${ }^{1}$ Department of Electrical and Electronics Engineering, University of Lagos, Lagos, Nigeria. \\ ${ }^{2}$ Computer and Electrical Engineering Department, Olabisi Onabanjo University, Ago-Iwoye, Ogun State, Nigeria.
}

\begin{tabular}{l} 
Article Info \\
\hline Article history: \\
Received July 21, 2017 \\
Revised May 19, 2018 \\
Accepted June 21, 2018
\end{tabular}

Keyword:

Device-to-Device (D2D)

$5 \mathrm{G}$ cellular networks continuous-time Markov chain (CTMC)

queueing model spectrum access

\begin{abstract}
Device-to-Device (D2D) communication has been considered a key enabling technology that can facilitate spectrum sharing in $4 \mathrm{G}$ and $5 \mathrm{G}$ cellular networks. In order to meet the high data rate demands of these new generation cellular networks, this paper considers the optimization of available spectrum resource through dynamic spectrum access. The utilization of continuous-time Markov chain (CTMC) model for efficient spectrum access in D2D-enabled cellular networks is investigated for the purpose of determining the impact of this model on the capacity improvement of cellular networks. The paper considers the use of CTMC model with both queueing and non-queueing cases called 13-Q CTMC and 6-NQ CTMC respectively with the aim of improving the overall capacity of the cellular network under a fairness constraint among all users. The proposed strategy consequently ensures that spectrum access for cellular and D2D users is optimally coordinated by designing optimal spectrum access probabilities. Numerical simulations are performed to observe the impact of the proposed Markovian queueing model on spectrum access and consequently on the capacity of D2D-enabled cellular networks. Results showed that the proposed 13-Q CTMC provide a more spectrumefficient sharing scheme, thereby enabling better network performances and larger capabilities to accommodate more users.
\end{abstract}

Copyright (C) 2018 Institute of Advanced Engineering and Science. All rights reserved.

Corresponding Author:

Abiodun Gbenga-Ilori

Department of Electrical and Electronics Engineering

University of Lagos, Lagos, Nigeria.

gbengailori@unilag.edu.ng

\section{INTRODUCTION}

Mobile data traffic, especially multimedia-rich services, are becoming available to more mobile users in recent years leading to an ever-increasing demand for higher data rate wireless access. Examples of present networks that demand higher data rates are the Long Term Evolution-Advanced (LTE-A) and Worldwide Interoperability for Microwave Access (WiMAX). There is also the next generation $5 \mathrm{G}$ network which will require even higher data rates in order to provide services to users. Due to bandwidth limitation, it is vital to utilize techniques which can achieve higher spectral efficiency. Traditionally, the cellular network operates on a centralized network topology which is not spectral efficient since it requires that mobile devices communicate through the base station even when they are in close proximity. As an alternative, D2D communication has been introduced to allow peer-to-peer transmission among mobile devices in close proximity, [1-3].

The advantages of allowing D2D communication underlay a cellular network is that it can increase area spectral efficiency, improve cellular coverage, reduce latency rate and also reduce energy consumption by mobile devices [4]. However, since D2D communication is lightly controlled by the base station, it poses a set of new challenges such as interference management and mode selection coordination. It is, therefore, necessary to efficiently and fairly share the spectrum resource among cellular users (CUs) and D2D users in order to take full advantage of the benefits of D2D communication and increase the overall capacity of the network.

A lot of research has been done in controlling interference in D2D communication underlaying cellular 
networks, [5-11]. A few papers have addressed this interference issue by controlling D2D access to the spectrum in a cellular network [12-15]. Various methods have also been used in the past for the analysis and design of D2D spectrum sharing. In [16], the authors used a Poisson point process (PPP) to design a spectrum sharing mode for D2D-enabled cellular networks. In [17], investigation of the throughput optimization problem in D2Dunderlaid cellular network while prioritizing cellular services was done. In [18], a mode selection algorithm to minimize outage probability and manage interference was proposed. In [11], a technique for determining the minimum distance between simultaneously operating D2D links in order to determine the minimum required signal-to-interference-plus-noise ratio (SINR) at all receivers in the network was introduced. A similar method was used in [7]. Some other papers used power control schemes for interference avoidance in the networks, [7, $8,13,19-21]$. Game theoretical approaches have also been used to control interference and for efficient resource allocation, [5], [22-25].

Although the existing dynamic spectrum access schemes have achieved some successes in enhancing spectrum efficiency, most of them do not address fairness in heterogeneous networks, [21]. Besides maximizing the overall spectrum utilization, a good spectrum-sharing scheme should also be able to achieve fairness among dissimilar users. The consequence of unfair resource allocation between dissimilar users may result in spectrum resource wastage or redundant allocation, [26]. CTMC-based models have been used before now for analyzing the performance of cognitive radio networks (CRNs). Most importantly, it has been used to model the spectrum access of primary and secondary users in the CRN in order to achieve an efficient, fair and flexible spectrum sharing, [27-33]. In [32], an M/D/1 priority queueing scheme was applied to evaluate the performance of CRNs. In [33], a primary-prioritized Markov approach was also used for dynamic spectrum access between secondary and primary users in CRNs. To the best of our knowledge, dynamic spectrum access schemes that can be used to improve the spectral efficiency of D2D-enabled cellular networks has not been well investigated.

Motivated by the successes of CTMC models for efficient and fair spectrum sharing among dissimilar users in CRNs, this paper proposes an optimized spectrum access strategy for combining CUs and D2D users in a cellular network. CTMC model is used with the aim of improving the overall capacity of the cellular network under a fairness constraint among users. The proposed strategy consequently ensures that there is no redundant allocation to a user while other users are in need of spectrum resource. Unlike previous approaches, spectrum access for D2D users is optimally coordinated by designing optimal spectrum access probabilities. Consequently, Markovian queueing and non-queueing models are used for dynamic spectrum access where the cellular spectrum sub-band is shared by a CU and 2 D2D users and later extended to the analysis of a general case with $N$ D2D users. The quality of service (QoS) constraint is defined by an SINR threshold that the CU should absolutely achieve. Hence depending on the channel state information received, the CU, D2D users or all $N+1$ users can transmit on the same frequency band. The computation time of this complex CTMC model consisting of one $\mathrm{CU}$ and $N$ D2D users is also quite low.

The key contributions of this paper can be summarized as follows:

- formulation of efficient spectrum access 6-NQ CTMC and 13-Q CTMC models to show the throughput gain possible in D2D-enabled cellular networks,

- proposal of a 13-Q CTMC model that ensures efficient and optimal spectrum access scheme for D2D users while protecting cellular users from intolerably high interference from D2D users,

- proposal of a 13-Q CTMC model that reduces the connection set-up time and thereby reducing the overall latency in the cellular network.

The remaining part of the paper is organized as follows. Section 2 presents the system model and assumptions. Section 3 presents the proposed Markovian non-queueing model and computation of the probability of co-transmission for multiple D2Ds and CU having SINR constraint. In Section 4, the Markovian queueing model is presented while the simulation studies are provided in Section 5. Finally, concluding remarks are provided in Section 6.

\section{SYSTEM MODEL}

In this paper, a dynamic spectrum access model is used in a cellular network where multiple D2D users are allowed to underlay licensed CUs. A network consisting of $N$ D2D links and $1 \mathrm{CU}$ device with licensed sub-band is considered. A sub-band is a frequency spectrum sub-allocated to a licensed cellular user. A cellular user owns a licensed sub-band which it can share with a number of D2D links.

Figure 1 shows the system diagram. The CU communicates solely through the base station using link $l$ while the two sets of D2D users communicate directly without the base station using links $D_{1}$ and $D_{2}$. It is 


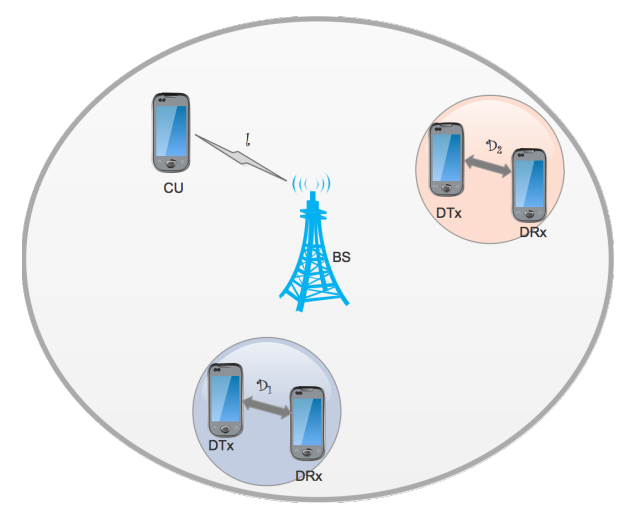

Figure 1. D2D-Enabled Cellular Network

assumed that all communication occurs in the same cell within the same channel. The paper also assumed that the D2D links share the uplink resource with the cellular user equipment. D2D communication is allowed as long as it does not cause SINR of the cellular link to drop below the required minimum. The SINR of the cellular link takes a higher priority.

The paper aims at determining the optimal spectrum access probabilities for each D2D link in the cellular network. If optimal coordination of D2D spectrum access can be guaranteed, then it is possible to achieve a good trade-off between spectrum efficiency and interference reduction. First, the spectrum access is modelled as a CTMC without queueing. In this case, if a D2D link does not meet the minimum requirement to underlay a cellular sub-band, it is dropped and has to start the process of spectrum search all over again. The disadvantage of this is that the D2D users spend more time and battery power is lost while searching for new spectrum. The spectrum access is later modelled as a CTMC with queueing where D2D links that do not meet present spectrum use requirements have the opportunity to queue up for a future time to access the spectrum instead of being dropped. This hopefully improves the network throughput while reducing communication setup time and battery power consumption.

The non-queueing CTMC spectrum access is modelled as a six-state CTMC while the queueing CTMC spectrum access is modelled as a thirteen-state CTMC. These state diagrams are used to compute the spectrum access probabilities $(\Pi)$ of being in each state. The average throughput $\mathcal{U}$ for each user in the cellular network is therefore computed as:

$$
\mathcal{U}=\Pi_{c} r_{c}+\Pi_{d 1} r_{d 1}+\cdots+\Pi_{d_{n}} r_{d_{n}}
$$

where the set $\Pi=\left\{\Pi_{c}, \Pi_{d 1}, \cdots, \Pi_{d_{n}}\right\}$ is the spectrum access probabilities of the cellular user $\mathcal{C}$ and set of $N$ D2D users $\mathcal{D}=\left[d_{1}, d_{2}, \cdots, d_{n}\right]$ and $\mathcal{U}$ is a function of $\Pi$ and the channel capacity of each user $r=$ $\left\{r_{c}, r_{d 1}, \cdots, r_{d_{n}}\right\}$. The channel capacity for a user $A$ operating in the spectrum band alone is

$$
r_{1}^{A}=W \log _{2}\left(1+\frac{P_{A} G_{A A}}{n_{0}}\right),
$$

and the channel capacity for user $A$ when it coexists with another user $B$ in the same spectrum band is

$$
r_{2}{ }^{A}=W \log _{2}\left(1+\frac{P_{A} G_{A A}}{n_{0}+\sum_{A \neq B} P_{B} G_{B A}}\right),
$$

where $W$ is the communication bandwidth, $n_{0}$ is the thermal noise power, $P_{A}$ and $P_{B}$ are the transmission power for users A and B respectively, and $G_{A A}$ is the channel gain for user A while $G_{B A}$ is the channel gain from user B's transmitter to user A's receiver.

Using the channel state information (CSI) gathered, the base station evaluates the spectrum utilization, computes the optimal access probabilities in different states and sends the results to the D2D link. The queueing model helps to determine the waiting period, if necessary, for each D2D link. Of course, if the waiting period is unacceptably long, the D2D link may choose to use another cellular link or even an unlicensed band. If a D2D user is transmitting and a CU arrives requesting the use of the channel and the minimum requirement is not met, the CU queue up and wait for the D2D user to complete transmission. However, if on arrival of the CU, there are $\mathrm{D} 2 \mathrm{D}$ users on the queue for spectrum use, the CU takes priority over the D2D users in the queue. 
Table 1. The Six States of the 6-NQ CTMC.

\begin{tabular}{|l|l|}
\hline State & Description \\
\hline 0 & No user in the spectrum \\
$C$ & CU in the spectrum \\
$D$ & One D2D user in the spectrum \\
1 & CU and one D2D user in the spectrum \\
2 & Both D2D users in the spectrum \\
3 & All users in the spectrum \\
\hline
\end{tabular}

\section{CELLULAR-PRIORITIZED NON-QUEUEING CTMC}

In this section, the dynamics of the system consisting of a CU and two D2D users is first modelled using a CTMC without queueing and later generalized to multiple D2D users. The probabilities involved in these transitions are also computed and used to derive the throughput that can be achieved in the cellular network.

\subsection{6-NQ CTMC}

In this section, it is assumed that when a D2D user requesting spectrum access appears, the base station determines if the D2D meet the minimum spectrum access requirements needed in the cellular network using the CSI. Otherwise, the D2D user is dropped and can either wait for a later time to try again, request for another cellular band or use an unlicensed spectrum band. The scenario is therefore modelled as a six-state non-queueing CTMC. First, it is assumed that a maximum of three users can use the single uplink frequency channel of the cellular user; $1 \mathrm{CU}$ and $2 \mathrm{D} 2 \mathrm{D}$ users. The paper later extends to a more general case of $N$-D2D users. In the non-queueing CTMC model, the CU's priority, in terms of spectrum access, is not so obvious. However, the base station gives the CU higher data rates compared to the D2D users. The spectrum access of the cellular and D2D users are modelled as independent Poisson process with arrival rates $\lambda_{c}$ and $\lambda_{d}$ respectively. The service times are assumed to be exponentially distributed with departure rates for cellular and D2D users denoted as $\mu_{c}$ and $\mu_{d}$ respectively. The six states of the non-queueing CTMC are described in Table 1. This six-state Markov chain is denoted by 6 -NQ CTMC for short.

The spectrum access process is shown in Figure 2. Assume at first that cellular band is idle, in which case the 6-NQ CTMC is in state 0 . In this case, there can be either an arrival of a cellular user $\mathcal{C}$ or a D2D user $d$. If any of these 2 users arrive, the 6-NQ CTMC transit to either state $C$ or $D$ with transition rates $\lambda_{c}$ and $\lambda_{d}$ respectively. If user $\mathcal{C}$ or $d$ complete service before any other user requests spectrum access, 6-NQ CTMC then transits to state 0 with departure rate $\mu_{c}$ and $\mu_{d}$ accordingly. However, if a second D2D arrives while the CU or the first D2D are in the spectrum, the 6-NQ CTMC transits to either 1 or 2 accordingly with rate $\lambda_{d}$. Once the $\mathrm{CU}$ or one of the D2D complete transmission, there is a transition to either state $C$ or $D$ with the departure rate of $\mu_{d}$. If both the CU and a D2D are in the spectrum and the second D2D requests a spectrum band, then the 6-NQ CTMC can either transit to state 3 from 2 with an arrival rate of $\lambda_{c}$ or transit to state 3 from state 1 with an arrival rate of $\lambda_{d}$. In all of the transitions described above, it has been assumed that no two D2D users can arrive or depart at exactly the same time. This assumption is justified for independent Poisson processes.

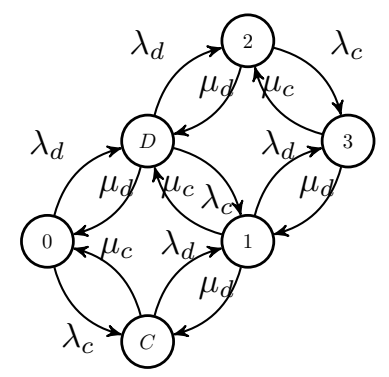

Figure 2. The Rate Diagram of 6-NQ CTMC 
The CTMC model is represented by $(\Pi, \mathcal{Q})$ where $\Pi=\left\{\Pi_{1}, \Pi_{2}, \cdots, \Pi_{n}\right\}$ is the state space while $\mathcal{Q}$ is the transition rate matrix and in our 6-NQ CTMC, $\Pi=\left\{\Pi_{0}, \Pi_{C}, \Pi_{D}, \Pi_{1}, \Pi_{2}, \Pi_{3}\right\}$.

$$
\mathcal{Q}=\left[q_{i j}\right],
$$

where $\mathcal{Q}$ in our 6 -NQ CTMC is given as the matrix:

$$
\mathcal{Q}=\left[\begin{array}{cccccc}
-\left(\lambda_{c}+\lambda_{d}\right) & \lambda_{c} & \lambda_{d} & 0 & 0 & 0 \\
\mu_{c} & -\left(\mu_{c}+\lambda_{d}\right) & 0 & \lambda_{d} & 0 & 0 \\
\mu_{d} & 0 & -\left(\mu_{d}+\mu_{c}+\lambda_{d}\right) & \lambda_{c} & \lambda_{d} & 0 \\
0 & \mu_{d} & \mu_{c} & -\left(\mu_{d}+\mu_{c}+\lambda_{d}\right) & 0 & \lambda_{d} \\
0 & 0 & \mu_{d} & 0 & -\left(\mu_{d}+\lambda_{c}\right) & \lambda_{c} \\
0 & 0 & 0 & \mu_{d} & \mu_{c} & -\left(\mu_{d}+\mu_{c}\right)
\end{array}\right]
$$

From the matrix shown above, $q_{i i}=-\sum_{j \neq i} q_{i j}$ and $0 \leq q_{i j}<\infty \forall i \neq j$. The balance equation to be solved is $\Pi \mathcal{Q}=0$ and $\sum \Pi_{n}=1$. Therefore the analysis of Figure 2 consists of the following system of equations;

$$
\left\{\begin{array}{l}
\Pi_{0}\left(\lambda_{c}+\lambda_{d}\right)=\Pi_{C} \lambda_{c}+\Pi_{D} \lambda_{d}, \\
\Pi_{C}\left(\mu_{c}+\lambda_{d}\right)=\Pi_{0} \mu_{c}+\Pi_{1} \lambda_{d}, \\
\Pi_{D}\left(\mu_{d}+\lambda_{c}+\lambda_{d}\right)=\Pi_{0} \mu_{d}+\Pi_{1} \lambda_{c}+\Pi_{2} \lambda_{d}, \\
\Pi_{1}\left(\mu_{d}+\mu_{c}+\lambda_{d}\right)=\Pi_{C} \mu_{d}+\Pi_{D} \mu_{c}+\Pi_{3} \lambda_{d}, \\
\Pi_{2}\left(\mu_{d}+\lambda_{c}\right)=\Pi_{D} \mu_{d}+\Pi_{3} \lambda_{c}, \\
\Pi_{3}\left(\mu_{c}+\mu_{d}\right)=\Pi_{1} \mu_{d}+\Pi_{2} \mu_{c}, \\
\quad \Pi_{0}+\Pi_{C}+\Pi_{D}+\Pi_{1}+\Pi_{2}+\Pi_{3}=1 .
\end{array}\right.
$$

Equation (5) represents the flow-balance at each of the six states and equation (6) represents the normalization equation that should satisfy a Markov chain with $\Pi_{n}$ being the steady state probabilities of being in a particular place where $n \in\{0, C, D, 1,2,3\}$. From these equations, the average throughput for the cellular $\left(\mathcal{U}_{c}\right)$ and each of the two D2D users $\mathcal{U}_{d_{1}}$ and $\mathcal{U}_{d_{2}}$ can be deduced as follows:

$$
\begin{gathered}
\mathcal{U}_{c}=\Pi_{C} r_{c}+\Pi_{1} r_{1}+\Pi_{3} r_{3}, \\
\mathcal{U}_{d 1}=\mathcal{U}_{d 2}=\Pi_{D} r_{d}+\Pi_{1} r_{1}+\Pi_{2} r_{2}+\Pi_{3} r_{3} .
\end{gathered}
$$

Total average throughput is therefore

$$
\mathcal{U}=\mathcal{U}_{c}+\mathcal{U}_{d 1}+\mathcal{U}_{d 2}
$$

\subsection{Generalized-NQ CTMC}

Our CTMC can be generalized to model the scenario with $1 \mathrm{CU}$ and $N$ D2D users as shown in the rate diagram of Figure 3 . In this case, the state space $\mathcal{S}_{N Q}$ has $2(N+1)$ states. $\mathcal{S}_{N Q}$ consists of a combination of the status of the $\mathrm{CU}$ and the $N$ D2D users and can be written as:

$$
\left(s_{C U}^{N Q}, s_{D 2 D}^{N Q}\right) \in \mathcal{S}_{N Q} \triangleq\left(\Phi_{C U}^{i d l e}, \Phi_{D 2 D}^{i d l e}\right) \bigcup\left(\Phi_{C U}, \Phi_{D 2 D}^{i d l e}\right) \bigcup\left\{\left(\Phi_{C U}, \Phi_{D 2 D}\right)\right\} \bigcup\left\{\left(\Phi_{C U}^{i d l e}, \Phi_{D 2 D}\right)\right\},
$$

where $\left(\Phi_{C U}^{i d l e}, \Phi_{D 2 D}^{i d l e}\right)$ is a state $(0,[0, \cdots, 0])$, in which there is no user requesting the spectrum. $\left(\Phi_{C U}, \Phi_{D 2 D}^{i d l e}\right)$ is a state $(1,[0, \cdots, 0])$ in which only the CU is using the spectrum. The set of states $\left\{\left(\Phi_{C U}, \Phi_{D 2 D}\right)\right\}$ represent all the states where a combination of $1 \mathrm{CU}$ and one or up to $N$ D2D users are in the spectrum. The set of states $\left\{\left(\Phi_{C U}^{i d l e}, \Phi_{D 2 D}\right)\right\}$ represent all the states where there is no CU but one or up to $N$ D2D users are in the spectrum.

If $q_{i j} \triangleq\left\{s_{i} \longrightarrow s_{j}\right\}$ denotes the transition from state $s_{i}$ to state $s_{j}$, then we can construct the matrix $\mathcal{Q}=\left[q_{i j}\right]$. For the state space $\mathcal{S}_{N Q}=\left[n_{0}, n_{1}, \cdots, n_{g}, \cdots, n_{N+1}\right]$ where $N+1$ is the number of users in the spectrum; CU or (and) D2D users, the number of transition states is given as $n=2(N+1)$ for $N$ D2D users. Therefore, $q\left\{\left[n_{0}, n_{1}, \cdots, n_{g}, \cdots, n_{N+1}\right] \longrightarrow\left[n_{0}, n_{1}, \cdots, 1-n_{g}, \cdots, n_{N+1}\right]\right\}=\mu_{g}$. We can also solve the stationary probability: $\Pi_{s n}=\left[\Pi_{s 1}, \cdots, \Pi_{s(2(N+1))}\right]$ using $\Pi \mathcal{Q}=0$ and $\sum_{n=1}^{2(N+1)} \Pi_{s_{n}}=1$. We can rewrite this as:

$$
\left[\begin{array}{c}
\mathcal{Q}^{T} \\
1_{1 \times(2(N+1))}
\end{array}\right]\left[\Pi^{T}\right]=\left[\begin{array}{c}
0_{(2(N+1)) \times 1} \\
1
\end{array}\right] .
$$




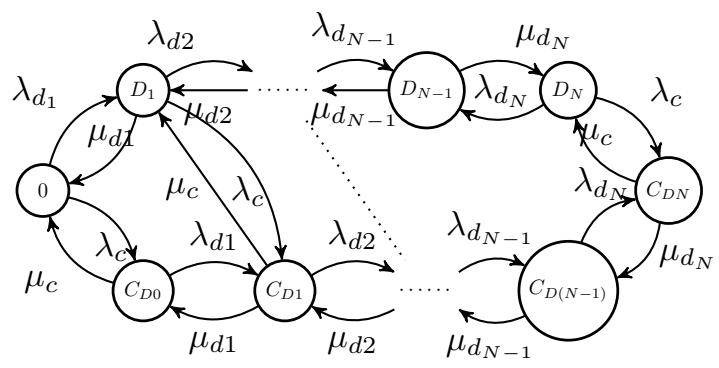

Figure 3. The Rate Diagram of the Generalized-NQ CTMC

\section{CELLULAR-PRIORITIZED QUEUEING CTMC}

In the previous section, D2D link that is unable to meet the QoS requirement of the network is dropped by the base station. This means that the D2D link is compelled to seek alternative means of communication. This has some disadvantages especially with respect to efficient usage of spectrum resources and delays in the network. In order to maximize the efficient use of these cellular channels, a concept was introduced where spectrum requests by D2D links that do not meet QoS of the network are queued in a buffer at the base station and the spectrum is immediately made available to D2D links on the queue without any time lapse in the usage of the licensed cellular bands. This way the overall communication set-up time for devices in the network is greatly reduced and the D2D user can conserve battery energy. First, this is modelled as a thirteen-state CTMC with queueing known as 13-Q CTMC and then later generalized in what is called the Generalized-Q CTMC.

\subsection{3-Q CTMC}

In this sub-section, the network is modelled to depict a situation where a D2D link request the use of a cellular spectrum and the base station, using the CSI, determines if the D2D link meets the minimum QoS requirement of the network. If it does, the D2D link is admitted into the channel. However, if it does not meet this requirement, it is admitted into a queue and can access the spectrum at a later time. The thirteen states of the 13-Q CTMC are described in Table 2 and the rate diagram is given in Figure 4.

Then the equation array governing the above system is given by:

$$
\begin{aligned}
& \begin{array}{l}
\Pi_{0}\left(\lambda_{c}+\lambda_{d}\right)=\Pi_{1} \lambda_{c}+\Pi_{2} \lambda_{d}, \\
\Pi_{1}\left(\mu_{c}+2 \lambda_{d}\right)=\Pi_{0} \mu_{c}+\Pi_{3} \lambda_{d}+\Pi_{6} \lambda_{d}, \\
\Pi_{2}\left(\mu_{d}+\lambda_{c}+2 \lambda_{d}\right)=\Pi_{0} \mu_{d}+\Pi_{4} \lambda_{d}+\Pi_{8} \lambda_{c}+\Pi_{12} \lambda_{d}, \\
\Pi_{3}\left(\mu_{d}+2 \lambda_{d}\right)=\Pi_{1} \mu_{d}+\Pi_{5} \lambda_{d}+\Pi_{11} \lambda_{d}, \\
\Pi_{4}\left(\mu_{d}+2 \lambda_{c}\right)=\Pi_{2} \mu_{d}+\Pi_{5} \lambda_{c}+\Pi_{9} \lambda_{c}, \\
\Pi_{5}\left(\mu_{d}+\mu_{c}\right)=\Pi_{3} \mu_{d}+\Pi_{4} \mu_{c}, \\
\Pi_{6}\left(\mu_{d}+\lambda_{d}\right)=\Pi_{1} \mu_{d}+\Pi_{7} \lambda_{c}, \\
\Pi_{7}\left(\mu_{d}\right)=\Pi_{6} \mu_{d}, \\
\Pi_{8}\left(\mu_{c}\right)=\Pi_{2} \mu_{c}, \\
\Pi_{9}\left(\mu_{c}\right)=\Pi_{2} \mu_{c}, \\
\Pi_{10}\left(\mu_{c}\right)=\Pi_{12} \mu_{c}, \\
\Pi_{11}\left(\mu_{d}\right)=\Pi_{3} \mu_{d}, \\
\Pi_{12}\left(\mu_{d}+\lambda_{c}\right)=\Pi_{2} \mu_{d}+\Pi_{10} \lambda_{c},
\end{array} \\
& \Pi_{0}+\Pi_{1}+\Pi_{2}+\Pi_{3}+\Pi_{4}+\Pi_{5}+\Pi_{6}+\Pi_{7}+\Pi_{8}+\Pi_{9}+\Pi_{10}+\Pi_{11}+\Pi_{12}=1 .
\end{aligned}
$$

Again the stationary probabilities can be solved by using $\Pi \mathcal{Q}=0$ and $\sum \Pi_{n}=1$ as shown in equations (12) and (13), and the total average throughput in the network can be determined from these equations. 
Table 2. The Thirteen States of the 13-Q CTMC.

\begin{tabular}{|l|l|}
\hline State & Description \\
\hline $0-(0,0)$ & No user in the spectrum \\
$1-(C, 0)$ & CU in the spectrum \\
$2-(D, 0)$ & 1 D2D user in the spectrum \\
$3-(1,0)$ & CU and 1 D2D user in the spectrum \\
$4-(2,0)$ & Both D2D users in the spectrum \\
$5-(3,0)$ & All 3 users in the spectrum \\
$6-\left(C, D_{w}\right)$ & CU in the spectrum, D2D user waiting \\
$7-(C, 2)$ & CU in the spectrum, 2 D2D users waiting \\
$8-\left(D, C_{w}\right)$ & 1 D2D user in the spectrum, CU waiting \\
$9-\left(2, C_{w}\right)$ & Both D2D users in the spectrum, CU waiting \\
$10-\left(D, 1_{w}\right)$ & 1 D2D user in the spectrum, 1 D2D and CU waiting \\
$11-\left(D, D_{w}\right)$ & 1 D2D user in the spectrum, 1 D2D waiting \\
$12-\left(1, D_{w}\right)$ & CU and 1 D2D user in the spectrum, 1 D2D waiting \\
\hline
\end{tabular}

\subsection{Generalized-Q CTMC}

We can generalize the CTMC with queueing model as:

$$
\left(s_{C U}^{Q}, s_{D 2 D}^{Q}\right) \in \mathcal{S}_{Q} \triangleq \mathcal{S}_{N Q} \bigcup \mathcal{S}_{W}
$$

where $\mathcal{S}_{Q}$ is the state space for the queueing model, $\mathcal{S}_{N Q}$ is as given in equation (10). The state space for the waiting incorporated is denoted by $\mathcal{S}_{W}$ and it is given as:

$$
\begin{array}{r}
\mathcal{S}_{W}=\left[\left\{\left(\Phi_{1 \text { iuser }}^{\text {in }}, \Phi_{1 u s e r}^{W}\right)\right\} \bigcup\left\{\left(\Phi_{1 u s e r}^{\text {in }}, \Phi_{2 u s e r s}^{W}\right)\right\} \bigcup \cdots \bigcup\left\{\left(\Phi_{1 u s e r}^{i n}, \Phi_{k-1 u s e r s}^{W}\right)\right\} \bigcup\right. \\
\left.\cdots \bigcup\left\{\left(\Phi_{2 \text { users }}^{\text {in }}, \Phi_{k-2 \text { users }}^{W}\right)\right\} \bigcup \cdots \bigcup\left\{\left(\Phi_{k-1 \text { users }}^{\text {in }}, \Phi_{1 \text { user }}^{W}\right)\right\}\right],
\end{array}
$$

where $k$ is the number of users in the spectrum; CU and D2D users inclusive. $\left(\Phi_{1 u s e r}^{\text {in }}, \Phi_{k-1 u s e r s}^{W}\right)$ means that 1 user is occupying the spectrum, CU or D2D, and the other $(k-1)$ users are waiting in the queue to use the spectrum.

The generator matrix $\mathcal{Q}=\left[q_{i j}\right]$ is again constructed for $s_{n}=\left[n_{0}, n_{1}, \cdots, n_{g}, \cdots, n_{k}\right]$ where $n=$ $N^{2}+4 N+1$ for $N$ D2D users. $q\left\{s_{n} \longrightarrow s_{n}^{w}\right\}$ denote transition that occurs when a user $j$ arrives given that the CSI does not support spectrum sharing with user $j$ at that time. The transition goes to $q\left\{s_{n}^{w} \longrightarrow s_{n}\right\}$ with the departure of some users and the accommodation of user $j$. The following equation array is then solved to obtain our stationary probabilities:

$$
\left[\begin{array}{c}
\mathcal{Q}^{T} \\
1_{1 \times\left(N^{2}+4 N+1\right)}
\end{array}\right]\left[\Pi^{T}\right]=\left[\begin{array}{c}
0_{\left(N^{2}+4 N+1\right) \times 1} \\
1
\end{array}\right]
$$

\section{NUMERICAL RESULTS}

In this section, the system performance of the 6-NQ CTMC and 13-Q CTMC spectrum access schemes are evaluated and analyzed in terms of the total throughput that can be achieved using each of them. The paper simulates a system with a cellular user and multiple D2D users arriving according to Poisson process with arrival rates $\lambda_{c}$ and $\lambda_{d}$ respectively. MATLAB is used to conduct the simulation experiments in order to determine the throughput in a cell using each of the two schemes discussed in sections III and IV. The goal is to compare the performance of the 6-NQ CTMC model with that of the 13-Q CTMC model in order to show the better performance of the proposed Markovian queueing model.

The following parameters were used: channel bandwidth $=5 \mathrm{MHz}$, UE transmitter power $=24 \mathrm{dBm}$, thermal noise per $\mathrm{MHz}=-114 d B$, receiver gain $=0 d B i, \lambda_{c}=1-20 s^{-1}, \lambda_{d}=1-20 s^{-1}, \mu_{c}=20 s^{-1}$, $\mu_{d}=25 \mathrm{~s}^{-1}$. The base station is located at the center of the $300 \mathrm{~m}$ radius cell and the CU and D2D users are distributed randomly around it. D2D links have a maximum distance of $20 \mathrm{~m}$. 


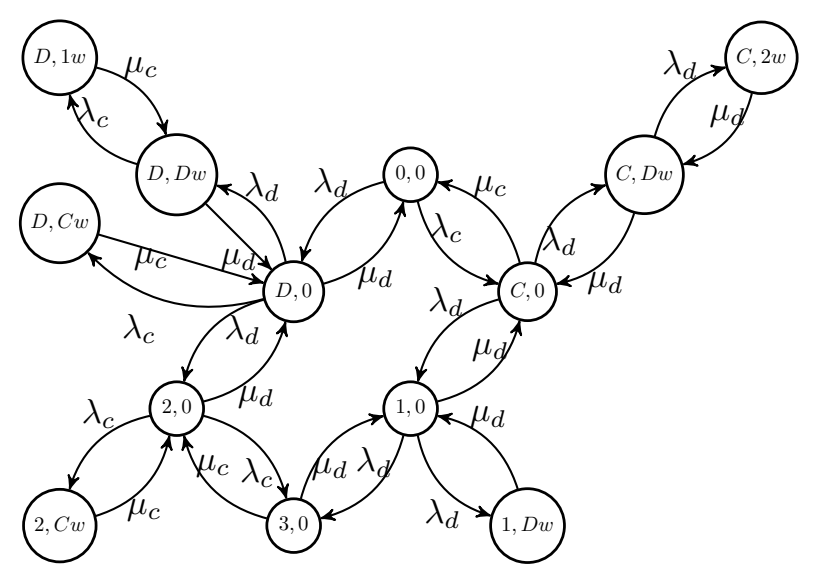

Figure 4. The Rate Diagram of 13-Q CTMC

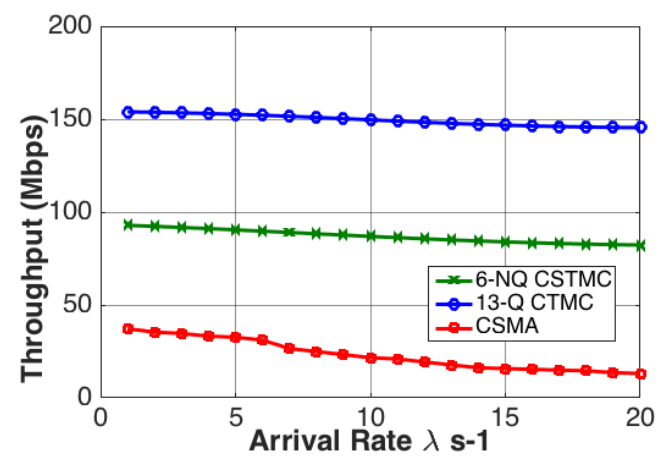

Figure 5. Average Throughput Achievable in Cell for 6-NQ CTMC and 13-Q CTMC

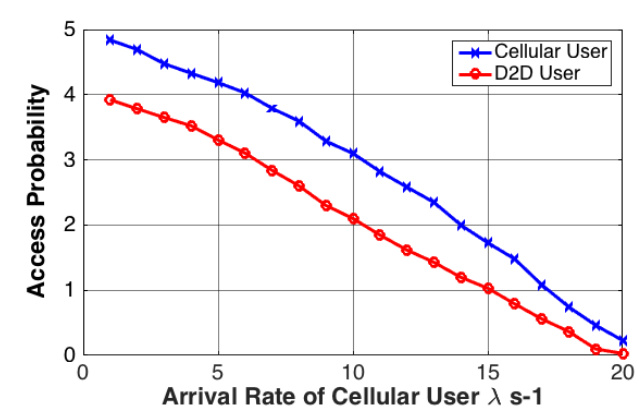

Figure 6. Optimal Access Probability for CU and D2D Users

The simulation is also used to determine the average waiting time in the entire system for both models. It is also used to determine the waiting time in the queue for the 13-Q CTMC model. This part of the simulation provides a way to assess the connection set-up time and overall latency in the system using the proposed queueing model. For determination of the waiting time in the systems and queue, the conventional method discussed in [34] is adopted. The performance of the proposed 13-Q CTMC model is also validated by comparing it with the existing non-persistent carrier sense multiple access (CSMA) spectrum access model reported in [35] to further show its superiority.

Figure 5 compares the throughput achievable by the proposed 13-Q CTMC model with that of the 6-NQ CTMC model and the existing non-persistent CSMA spectrum access technique. It can be shown, by comparing throughput achievable from the three spectrum access schemes, that 13-Q CTMC has the highest throughput while CSMA has the lowest throughput. The poor performance of CSMA is due to the collision rate and inefficient random waiting time of this scheme. However, by controlling the access probabilities of D2D users in both CTMC schemes, it was possible to accommodate more traffic and greatly increase the throughput. The 13-Q CTMC model was able to achieve further increase in throughput because queueing UEs, instead of rejecting requests, improved the overall throughput of the network. Generally, there is a throughput degradation for all models as $\lambda$ increases. This is as a result of interference that may occur in the network.

Though there is a general degradation in throughput as $\lambda$ increases, yet a slower rate of degradation was noticed in the proposed 13-Q CTMC model with a degradation of $0.58 \%$ in throughput when $\lambda$ was increased from 1 to 4 and $5.53 \%$ degradation in throughput when $\lambda$ was increased from 1 to 20 . In the 6-NQ CTMC model, when $\lambda$ was increased from 1 to 4 , the network experienced a $1.9 \%$ degradation in throughput and when $\lambda$ was increased from 1 to $20,11.43 \%$ degradation in throughput was experienced in the network. The CSMA performed very poorly with degradation of $10.7 \%$ as $\lambda$ increased from 1 to 4 and degradation of $65 \%$ as $\lambda$ increased from 1 to 20 . Therefore the 13-Q CTMC was able to achieve the highest throughput and also has the best access scheme because it performed best with an increase in arrival rate. 


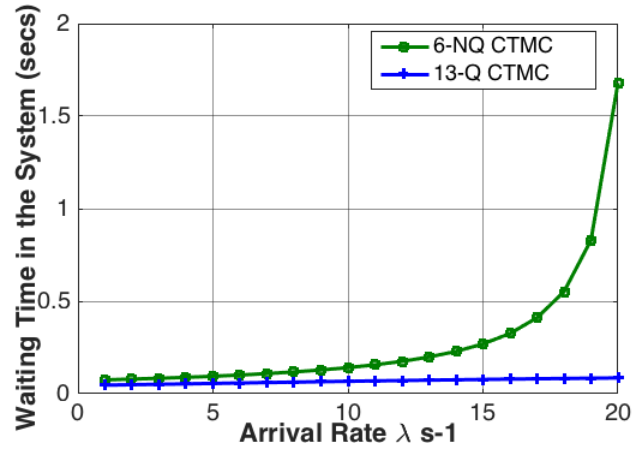

Figure 7. Waiting Time in the System for 6-NQ CTMC and 13-Q CTMC

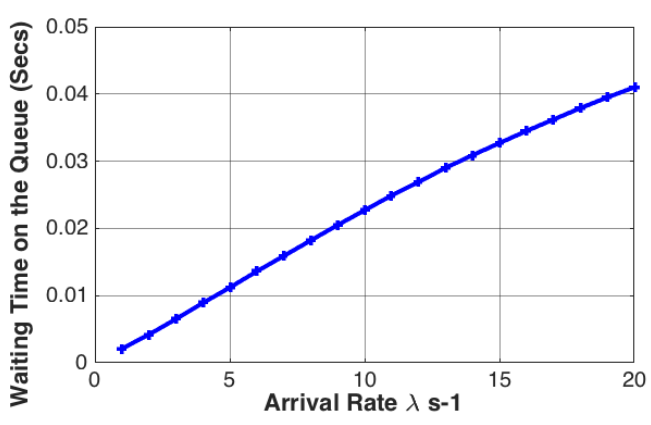

Figure 8. Waiting Time on the Queue for 13-Q CTMC

Figure 6 shows the average access probability of D2D and cellular users. The access probability of the cellular user decreases as arrival rate of cellular users increases. The arrival rate of the cellular user also causes a reduced access probability for the D2D user. However, the access probability of the cellular user is still more than that of the D2D user because of the priority given to the cellular user since its arrival rate is fixed at $20 s^{1}$.

The service time in the network for our 6-NQ CTMC was also compared with that of the 13-Q CTMC. It can be shown from Figure 7 that the waiting time in both models is comparably close when the number of arrivals, $\lambda$, is less than 4 users per seconds with 13-Q CTMC model slightly better than the 6-NQ CTMC model. However, as the number of arrivals per second increases, the waiting time in the system increases exponentially in the 6-NQ CTMC model. It is seen that the 13-Q CTMC was able to perform better in that it is able to tolerate interference in the system despite the inclusion of queueing time in this model.

Figure 8 shows the waiting time on the queue for the 13-Q CTMC. We see that even with the queue, users will still have access to the system faster than using the 6-NQ CTMC. The proposed 13-Q CTMC, therefore, showed better performances and larger capabilities to accommodate more users. It also offers a more efficient spectrum utilization compared to 6-NQ CTMC.

\section{CONCLUSION}

In the paper, a Markovian-queueing approach is proposed for optimizing the use of cellular spectrum resource through dynamic spectrum access. This is highly necessary in order to meet the high data rate demands of $4 \mathrm{G}$ and $5 \mathrm{G}$ cellular networks. The use of a Markovian-queueing model known as 13-Q CTMC is proposed for underlaying D2D users in a cellular bandwidth in order to optimize the use of spectrum resource and increase throughput in the network. A Markovian-queueing model is chosen because of the successes of CTMC models in achieving efficient and fair spectrum sharing in heterogeneous networks. The proposed 13-Q CTMC queueing model is compared with the 6-NQ CTMC model that does not support queueing and the existing non-persistent CSMA spectrum access scheme. Simulation results showed that the proposed Markovian-queueing model is more efficient in the use of limited spectrum resource and also yielded better throughput in the network compared to the other two spectrum access techniques. The 13-Q CTMC model ensures efficient and optimal spectrum access scheme for D2D users while protecting cellular users from intolerably high interference from D2D users. Compared with the other spectrum access techniques, the 13-Q CTMC model showed a considerable reduction in the connection set-up time and thereby reducing the overall latency in the cellular network.

\section{ACKNOWLEDGMENT}

The first author will like to acknowledge the support of the Alexander von Humboldt Foundation for financing her post-doctoral research stay at the Ruhr-Universität Bochum, Germany.

\section{REFERENCES}

[1] A. Krishna, A. Chakravarthy, and A. Sastry, "A Hybrid Cryptographic System for Secured Device to Device Communication," International Journal of Electrical and Computer Engineering (IJECE), vol. 6, no. 6, pp. 2962-2970, December 2016. 
[2] A. Asadi, Q. Wang, and V. Mancuso, "A Survey on Device-to-Device Communication in Cellular Networks," IEEE Communications Surveys and Tutorials, vol. 2014, no. 4, pp. 1801-1819, April 2014.

[3] A. Asadi and V. Mancuso, "Network-assisted Outband D2D-clustering in 5G Cellular Networks: Theory and Practice," IEEE Transactions on Mobile Computing, vol. PP, no. 99, pp. 1-15, October 2016.

[4] L. Song, D. Niyato, Z. Han, and E. Hossain, "Game-Theoretic Resource Allocation Methods for Deviceto-Device Communication,” IEEE Wireless Communication, vol. 21, no. 3, pp. 136-144, June 2014.

[5] Y. Xiao, K. Chen, C. Yuen, and L. DaSilva, "Spectrum Sharing for Device-to-Device Communications in Cellular Networks: A Game Theoretic Approach," in Proc. IEEE Conference on Dynamic Spectrum Access Networks (DYSPAN), McLean, VA, USA, 2014.

[6] D. Feng, L. Lu, Y. Yuan-Wu, G. Y. Li, G. Feng, and S. Li, "Device-to-Device Communications in Cellular Networks," IEEE Communications Magazine, vol. 52, no. 4, pp. 49-55, April 2014.

[7] D. Feng, L. Lu, Y. Yuan-Wu, G. Y. Li, S. Li, and G. Feng, "Device-to-Device Communications Underlaying Cellular Networks," IEEE Trans. on Communications, vol. 61, no. 8, pp. 3541-3551, Aug. 2013.

[8] P. Jänis, C. Yu, K. Doppler, C. Ribeiro, C. Wijting, K. Hugl, O. Tirkkonen, and V. Koivunen, "Device-toDevice Communication Underlaying Cellular Communication Systems," International Journal of Communications, Network and System Sciences, no. 3, pp. 169-178, June 2009.

[9] I. Iskandar, I. Setyawan, and H. Nuraini, "Inter-cell interference management technique for multi-cell lte-a network," International Journal of Electrical and Computer Engineering (IJECE), vol. 7, no. 5, p. 2696, 2017.

[10] T. Peng, Q. Lu, H. Wang, S. Xu, and W. Wang, "Interference Avoidance Mechanisms in the Hybrid Cellular and Device-to- Device Systems," in Proc. of IEEE 20th International Symposium on Personal, Indoor and Mobile Radio Communications, Tokyo, Japan, Sept. 2009, pp. 617-621.

[11] M. Meibergen, "Device-to-Device Communications Underlaying a Cellular Network," Master's thesis, Delft University of Technology, Netherlands, 2011.

[12] K. Huang, V. Lau, and Y. Chen, "Spectrum Sharing Between Cellular and Mobile Ad Hoc Networks: Transmission-Capacity Trade-Off," IEEE Journal on Selected Areas in Communications, vol. 27, no. 7, pp. 1256-1267, 2009.

[13] C. Yu, K. Doppler, C. Ribeiro, and O. Tirkkonen, "Resource Sharing Optimization for Device-to-Device Communication Underlaying Cellular Networks," IEEE Trans. on Wireless Communications, vol. 10, no. 8, pp. 2752-2763, 2011.

[14] B. Kaufman, J. Lilleberg, and B. Aazhang, "Spectrum Sharing Scheme Between Cellular Users and AdHoc Device-to-Device Users," IEEE Trans. on Wireless Communications, vol. 12, no. 3, pp. 1038-1049, March 2013.

[15] H. Esmat, M. Elmesalawy, and I. Ibrahim, "Adaptive Resource Sharing Algorithm for Device-to-Device Communications Underlaying Cellular Networks," IEEE Communications Letters, vol. 20, no. 3, pp. 530533, January 2016.

[16] X. Lin, J. Andrews, and A. Ghosh, "Spectrum Sharing for Device-to-Device Communication in Cellular Networks," IEEE Trans. on Wireless Communications, vol. 12, no. 13, pp. 6727-6740, 2014.

[17] C. Yu, O. Tirkkonen, K. Doppler, and C. Ribeiro, "Power optimization of device-to-device communication underlaying cellular communication," in Proc. 2009 IEEE International Conference on Communications (ICC), Dresden, Germany, 2009.

[18] H. Min, W. Seo, J. Lee, and D. Hong, "Reliability Improvement Using Receive Mode Selection in the Device-to-Device Uplink Period Underlaying Cellular Networks," IEEE Trans. on Wireless Communications, vol. 10, no. 2, pp. 413-418, February 2011.

[19] N. Lee, Y. Lin, J. Andrews, and R. H. Jr., "Power Control for D2D Underlaid Cellular Networks: Modeling, Algorithms, and Analysis," IEEE Journal on Selected Areas in Communications, vol. 33, no. 1, pp. 1-13, November 2014.

[20] R. AliHemmati, B. Liang, M. Dong, G. Boudreau, and A. Seyedmehdi, "Multi-channel power allocation for device-to-device communication underlaying cellular networks," in Proc. 20166 IEEE International Conference on Acoustics, Speech and Signal Processing (ICASSP), Shanghai, China, 2016.

[21] J. Chen, C. Liu, H. Li, X. Li, H. Wang, and S. Li, "A Categorized Resource Sharing Mechanism for Device-to-Device Communications in Cellular Networks," Mobile Information Systems, vol. 2016, no. ID 5894752, pp. 1-10, October 2016.

[22] Y. Liu and S. Feng, "Interference Pricing for Device-to-Device Communications," in Proc. of IEEE ICC 2014 Wireless Communication Symposium, Sydney, Australia, June 2014.

[23] P. Yuan and G. Bi, "Resource Allocation in D2D-Enabled Cellular Networks Using Hierarchical Game," in 
Proc. Third International Conference on Future Generation Communication Technologies (FGCT), London, UK, 2014.

[24] A. Gbenga-Ilori and S. Aydin, "Channel Access in D2D Multiuser Networks: A Game Theoretical Approach," in Proc. 20th International ITG Workshop on Smart Antennas (WSA), Munich, Germany, 2016.

[25] F. Boabang, H. Nguyen, Q. Pham, and W. Hwang, "Network-Assisted Distributed Fairness-Aware Interference Coordination for Device-to-Device Communication Underlaid Cellular Networks," Mobile Information Systems, vol. 2017, no. ID 1821084, pp. 1-11, January 2017.

[26] H. Shi, "Fairness and Resource Allocation in Device-to-Device Wireless Regional Area Network," Ph.D. dissertation, Delft University of Technology, Netherlands, 2014.

[27] M. Elalem and L. Zhao, "Throughput Maximization Based on Optimal Access Probabilities in Cognitive Radio System,” Journal of Communications and Networks, vol. 5, no. 3, pp. 204-210, 2013.

[28] M. Amini, A. Mirzavandi, and M. Rezaei, "Discrete Markov Chain Based Spectrum Sensing for Cognitive Radio," International Journal of Electrical and Computer Engineering (IJECE), vol. 5, no. 2, pp. 297-303, April 2015.

[29] U. Mia, L. M. Boulahia, M. Esseghir, and D. Gaiti, "A Continuous Time Markov Model for Unlicensed Spectrum Access," in Proc. IEEE 7th International Conference on Wireless and Mobile Computing, Networking and Communications (WiMob), Shanghai, China, 2011.

[30] Y. Zhao, S. Jin, and W. Yue, "A novel spectrum access strategy with alpha Retry policy in cognitive radio networks: A queueing-based analysis," Journal of Communications and Networks, vol. 16, no. 22, pp. 193-201, April 2014.

[31] H. Ngallemo, W. Ajib, and H. Elbiaze, "Dynamic spectrum access analysis in a multi-user cognitive radio network using Markov chains," in Proc. 2012 International Conference on Computing, Networking and Communications (ICNC), Maui, USA, 2012.

[32] I. Suliman and J. Lehtomki, "Queueing Analysis of Opportunistic Access in Cognitive Radios," in Proc. of IEEE International Workshop on Cognitive Radio and Advanced Spectrum Management (CogRAT), 2009, pp. $153-157$.

[33] B. Wang, Z. Ji, and K. RayLiu, "Primary-Prioritized Markov Approach for Dynamic Spectrum Access," IEEE Trans. on Wireless Communications, vol. 8, no. 4, pp. 1854-1856, 2009.

[34] G. Bolch, S. Greiner, H. de Meer, and K. Trivedi, “Queueing Networks and Markov Chains," in John Wiley and Sons Inc., Second Edition, New Jersey, USA, 2006.

[35] H. Takagi and L. Kleinrock, "Packet Switching in Radio Channels: Part I: Carrier Sense Multiple-Access Modes and Their Throughput-Delay Characteristics," IEEE Transaction on Communications, vol. 23, no. 12, pp. 1400-1416, November 1975.

\section{BIOGRAPHIES OF AUTHORS}

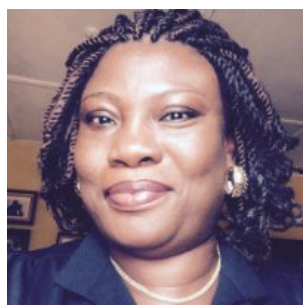

Abiodun Gbenga-Ilori is a Senior Lecturer in the Department of Electrical and Electronics Engineering, University of Lagos where she has been a faculty member since 2003. Dr. Gbenga-Ilori completed her Ph.D. in Communication Engineering at the University of Ilorin and her undergraduate studies at the University of Ibadan. Her research interests are interference mitigation in communication networks and optimisation of frequency spectrum use. She has over 20 publications in these areas. In recent years, she has focused on the game theoretic approach to communication network planning and Device-to-Device (D2D) for 5G networks. She is an Alexander von Humboldt fellow and has collaborated actively with researchers internationally especially in Germany. She is also a reviewer for a few journals including IEEE Vehicular Technology Transaction and a registered member of the Council of Registered Engineers of Nigeria (COREN).

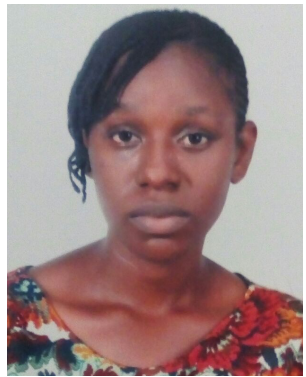

Olufunmilayo Sanusi Olufunmilayo Sanusi received her B.Sc. in Computer Engineering from Olabisi Onabanjo University and her M.Sc. in Communication Engineering from the University of Lagos. She is currently pursuing a Ph.D. in Communication Engineering at the University of Lagos. Her major research interests include interference analysis in radio communication networks and coexistence of heterogeneous devices in communication networks. She is a registered member of the Council of Registered Engineers of Nigeria (COREN). 